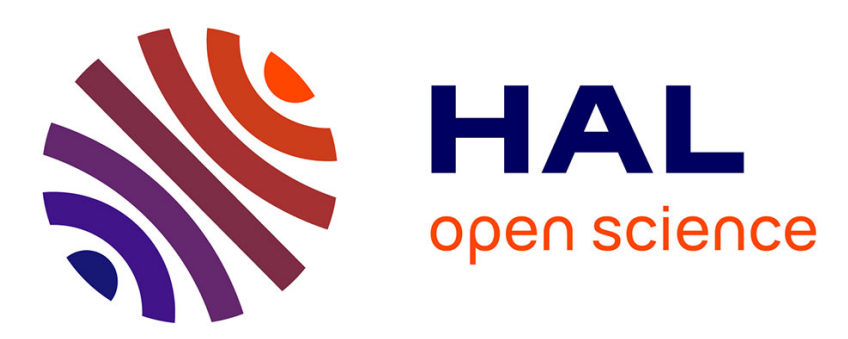

\title{
Association entre régimes végétariens et pathologies chroniques: une approche épidémiologique
}

\author{
Luc Dauchet, Yu Jin Jung
}

\section{To cite this version:}

Luc Dauchet, Yu Jin Jung. Association entre régimes végétariens et pathologies chroniques: une approche épidémiologique. Cahiers de Nutrition et de Diététique, 2019, 54, pp.318 - 325. 10.1016/j.cnd.2019.07.004 . hal-03488402

\section{HAL Id: hal-03488402 https://hal.science/hal-03488402}

Submitted on 21 Dec 2021

HAL is a multi-disciplinary open access archive for the deposit and dissemination of scientific research documents, whether they are published or not. The documents may come from teaching and research institutions in France or abroad, or from public or private research centers.
L'archive ouverte pluridisciplinaire HAL, est destinée au dépôt et à la diffusion de documents scientifiques de niveau recherche, publiés ou non, émanant des établissements d'enseignement et de recherche français ou étrangers, des laboratoires publics ou privés.

\section{다)(1) $(5$}

Distributed under a Creative Commons Attribution - NonCommerciall 4.0 International 


\title{
Association entre régimes végétariens et pathologies chroniques: une approche épidémiologique (Association between vegetarian diets and chronic diseases: an epidemiological approach)
}

\section{Luc Dauchet $^{1}$, Yu jin Jung ${ }^{1}$}

${ }^{1}$ Univ. Lille, Inserm, CHU Lille, Institut Pasteur de Lille, U1167 - RID-AGE - Facteurs de risque et déterminants moléculaires des maladies de l'âge, F-59000 Lille, France

\begin{abstract}
Interest in vegetarian diets is growing. The proportion of vegetarians in the population is about $2 \%$. Adopting a vegetarian diet has potentially beneficial health effects but, if poorly managed, may not allow to cover all nutritional needs. Epidemiological studies conducted on specific populations, including mostly well-informed vegetarians (Oxford and Adventists cohorts), have shown reduced risk of obesity, mortality and vascular disease among vegetarians. These results suggest that wellmanaged vegetarian diet is beneficial in terms of health. However, the risks associated with poorly managed diets are insufficiently assessed in these epidemiological studies, particularly in the elderly and growing children and adolescents. The additional benefit of a vegetarian diet compared to an omnivorous diet of good nutritional quality remains to be assessed.
\end{abstract}

Résumé

L'intérêt pour les régimes végétariens est croissant. La proportion de végétariens dans la population est d'environ $2 \%$. L'adoption d'un régime végétarien a des effets potentiellement bénéfiques pour la santé mais pourrait, s'il est mal conduit, ne pas permettre de couvrir l'ensemble des besoins nutritionnels. Les études épidémiologiques conduites sur des populations spécifiques, incluant des végétariens majoritairement bien informés (cohortes d'Oxford et des adventistes) retrouvent une diminution du risque d'obésité, de mortalité et de maladies vasculaires. Ces résultats suggèrent un bénéfice du régime végétarien bien conduit en termes de santé. Cependant, les risques liés à des régimes mal conduits sont insuffisamment évalués dans ces études épidémiologiques, notamment chez les personnes âgées et les enfants et les adolescents en période de croissance. Le bénéfice supplémentaire apporté par un régime végétarien en comparaison d'un régime omnivore de bonne qualité nutritionnelle reste à évaluer. 


\section{Introduction}

L'intérêt de la population pour les régimes végétariens est croissant pour des raisons éthiques, des préoccupations environnementales et de santé. La consommation de régimes végétariens peut induire des risques d'insuffisance d'apports nutritionnels mais peut aussi avoir un impact bénéfique sur la santé, en particulier pour la prévention de l'obésité, des maladies cardiovasculaires et des cancers (1).

\subsection{Définition des régimes végétariens}

Un régime végétarien peut être défini comme un régime excluant la viande et le poisson. En pratique, il faudrait plutôt évoquer « les régimes végétariens » au pluriel. Il existe plusieurs types de régimes restreints en produits d'origine animale que l'on pourrait classer en fonction du type d'aliments exclus (2).

- Les «flexitariens » ou " semi-végétariens » ont une consommation occasionnelle de viande et de poisson ;

- Les « pesco-végétariens » consomment du poisson mais excluent les autres viandes.

Les flexitariens et les pesco-végétariens ne font pas partie des végétariens au sens strict. Le terme végétarien englobe les régimes suivants :

- Les lacto/ovo-végétariens ne consomment pas de viande mais consomment d'autres produits d'origine animale. Ils consomment des œufs et/ou du lait ;

- Les végétaliens excluent tout aliment d'origine animale de leur alimentation.

Les conséquences en termes de bénéfice et de risque de ces régimes diffèrent. Plus les régimes sont restrictifs, plus les risques d'insuffisance d'apports sont importants. Ce sont essentiellement les régimes végétariens qui ont été évalués dans les études épidémiologiques et de façon moins fréquente les régimes végétaliens.

\subsection{Proportion de la population consommant un régime végétarien}

En France, la prévalence des personnes déclarant suivre un régime végétarien a été estimée à $0,5 \%$ (IC95\% [0,3\%-0,8\%]) dans l'étude INCA 2 (2006-2007). Cette prévalence était en augmentation dans l'étude INCA 3 (2014-2015) à 1,8\% (IC95\% [1,1\%-2,9\%]) (3). Dans l'étude INCA 3, la prévalence des personnes déclarant consommer un régime végétalien était marginale à $0,1 \%$ (soit 2 personnes parmi les participants) (3). L'étude Nutrinet est une cohorte de volontaires recrutés sur internet. Elle n'est pas représentative de la population générale et l'on peut suspecter que les végétariens motivés par les questions d'alimentation soient sur-représentés dans cette cohorte. Néanmoins, la prévalence de $2,5 \%$ de végétariens retrouvée dans cette étude conforte les résultats de l'étude INCA 3. La prévalence du régime végétalien était de $0,8 \%$ dans la cohorte Nutrinet (4). Ce chiffre est probablement surévalué par rapport aux données des études INCA, qui elles, sont issues d'un échantillon représentatif de la population française. La comparaison des résultats des études INCA 2 et INCA 3 suggère une augmentation de la proportion de végétariens en France mais cette proportion reste faible et la proportion de végétaliens reste encore marginale. L'évolution de ces prévalences est à surveiller. 
Au niveau international, l'étude National Health and Nutrition Examination Survey (NHANES) (4) a retrouvé une proportion de $2,3 \%$ de végétariens aux Etats-Unis, une prévalence proche de ce qui est observé en France. Des proportions plus importantes de végétariens ont été retrouvées en Allemagne (6,1\% des femmes et $2,5 \%$ des hommes âgés de 18 à 79 ans)(5) et en Inde (de 10 à $62 \%$ en fonction des régions) $(6,7)$.

\subsection{Evolution des recommandations et des points de vue des sociétés savantes}

Les recommandations concernant la consommation de viande, et les positions des sociétés savantes concernant les régimes végétariens, ont progressivement évolué. A titre d'exemple, l'American Dietetic Association (ADA) émettait des « doutes raisonnables sur l'adéquation nutritionnelle et les bénéfices d'un régime végétarien » (traduit de l'anglais) en $1980(8,9)$. En 1993, le régime végétarien lui apparaissait comme une alternative équivalente au régime conventionnel. Ses conclusions étaient: " un régime végétarien bien conduit peut permettre d'atteindre les recommandations et peut être un régime alternatif favorable à la santé " (traduit de l'anglais) (10). En 2016, les conclusions de l'ADA suggéraient que le régime végétarien serait supérieur au régime omnivore conventionnel en termes de bénéfices pour la santé (11) (« en comparaison à un régime non végétarien, le régime végétarien peut apporter une protection contre de nombreuses maladies chroniques ") et soulignaient également les bénéfices potentiels pour l'environnement. La position de cette société savante est citée à titre d'illustration et ne peut pas être considérée comme représentative de l'ensemble de la communauté scientifique. En France, le régime végétarien ne fait pas l'objet d'une recommandation. Cependant, dans l'avis relatif à la révision des repères alimentaires pour les adultes du futur Programme National Nutrition Santé (PNNS) 2017-2021 (12), il est conseillé de limiter la consommation de viande rouge à $500 \mathrm{~g}$ par semaine et la consommation de charcuterie à $150 \mathrm{~g}$ par semaine. II n'y a plus de recommandation de consommation minimale de viande. De plus, la recommandation en matière de produits laitiers est passée de 3 à 2 portions par jour. Ces recommandations tendraient à une diminution de la consommation des produits d'origine animale.

\section{Bénéfices / risques}

\subsection{Couverture des besoins nutritionnels}

L'exclusion totale ou partielle d'aliments d'origine animale pourrait conduire à des insuffisances d'apports en certains macronutriments ou micronutriments. Un régime végétarien pourrait à ce titre constituer un potentiel risque pour la santé.

Les produits d'origine animale sont une source importante de protéines. On pourrait donc faire I'hypothèse qu'un régime végétarien se traduirait par une insuffisance d'apport en protéines. En particulier, un apport en protéines issues de sources non variées et provenant essentiellement des produits céréaliers pourrait conduire à un apport insuffisant en lysine. Mais si les sources de protéines sont variées, un régime à base d'aliments d'origine végétale peut apporter les apports en acides aminés essentiels nécessaires au bon fonctionnement de l'organisme d'un adulte sain (13).

D'autres insuffisances d'apports pourraient potentiellement être induites par un régime végétarien et plus particulièrement par un régime végétalien (11). Les apports en acide gras $n-3$, en zinc et en iode sont moins importants dans les régimes végétariens et végétaliens. Cependant ces faibles apports peuvent être compensés par l'augmentation de la consommation de certains aliments tels que les graines, les noix et le sel iodé. Une supplémentation en iode peut être nécessaire chez les femmes en âge de procréer. 
1 Les végétariens consomment généralement autant de fer que les non végétariens, mais la 2 biodisponibilité du fer non héminique d'origine végétale est moindre, conduisant à un plus faible 3 stockage du fer et un taux de ferritine plus bas. Ce phénomène est partiellement compensé par une 4 augmentation de la capacité d'absorption du fer quand la ferritine diminue.

Les apports en calcium sont généralement supérieurs ou égaux aux recommandations chez les personnes ayant un régime ovo/lacto-végétarien, en revanche, ils sont généralement plus faibles chez les végétaliens. Une augmentation du risque de fractures tous types a été constatée chez les végétaliens dans l'étude EPIC-Oxford (14). Des apports insuffisants en vitamine D ont été constatés chez les végétariens pour lesquels une supplémentation est souvent nécessaire. Cependant, une supplémentation est également souvent nécessaire chez les personnes ayant un régime omnivore.

Enfin, la vitamine B12 n'est pas retrouvée dans les produits d'origine végétale et une supplémentation est nécessaire afin d'éviter une carence.

Ce paragraphe n'est pas exhaustif sur l'ensemble des impacts nutritionnels des régimes végétariens. D'une manière générale, les besoins nutritionnels peuvent être généralement couverts par un régime bien conduit. Cependant, il est nécessaire d'avoir un régime correctement planifié voire un suivi nutritionnel, en particulier pour les personnes adoptant un régime végétalien. Il est à noter, à titre d'illustration, que les avis favorables de I'ADA portent sur des régimes qualifiés de "welldesigned vegetarian diets» (11).

\subsection{Bénéfices attendus}

L'adoption d'un régime végétarien conduit à la diminution de consommation de produits d'origine animale dont certains peuvent être associés à une augmentation du risque de développement de pathologies chroniques. A l'inverse, l'adoption d'un régime végétarien conduit à l'augmentation de consommation de produits d'origine végétale dont certains pourraient avoir un impact favorable sur la santé. Sont présentés dans ce chapitre les bénéfices potentiels de la diminution de la consommation de certains produits d'origine animale, et de l'augmentation de la consommation de certains produits d'origine végétale.

\subsubsection{Viandes}

La viande rouge a été classée comme carcinogène probable (Groupe 2A) par le Centre international de recherche sur le cancer (CIRC). Une consommation supplémentaire de viande rouge de $120 \mathrm{~g}$ par semaine augmenterait de $2 \%$ le risque de décès par cancer (15) et serait associée à une augmentation du risque de mortalité d'environ $15 \%$ pour une portion supplémentaire de $120 \mathrm{~g}$ par jour. La charcuterie a été classée comme carcinogène (groupe 1) par le CIRC (16). Une augmentation de la consommation de charcuterie de 50g par semaine serait associée à une augmentation de $4 \%$ du risque de mortalité par cancer (17). La consommation de viande rouge et de charcuterie semble être particulièrement associée à certaines localisations de cancer. Par exemple, une augmentation de la consommation de viande rouge de 100g par jour serait associée à une augmentation de $43 \%$ du risque de cancer du pancréas (chez l'homme), du cancer du côlon proximal (11\%), du cancer du côlon distal (22\%) et du cancer du rectum (23\%) (18). Une augmentation de la consommation de 50g par jour serait associée à une augmentation de $21 \%$ du risque de cancer du pancréas (chez l'homme) et une augmentation de $18 \%$ du risque de cancer colorectal (15).

De plus, une augmentation de la consommation de viande rouge de $100 \mathrm{~g}$ par jour serait également associée à une augmentation de $13 \%$ du risque de survenue de diabète de type 2 (19).

La consommation de viande serait associée à une augmentation du risque de mortalité cardiovasculaire dans les pays occidentaux, mais cette association n'a pas été retrouvée en Asie 
$(17,20)$. En effet, une consommation de $120 \mathrm{~g}$ de viande par semaine serait associée à une augmentation de $1 \%$ du risque de mortalité cardiovasculaire et une augmentation de la consommation de charcuterie de $50 \mathrm{~g}$ par semaine serait associée à une augmentation de $2 \%$ de ce risque.

5

La viande rouge et la charcuterie sont une composante importante du régime occidental (western diet) qui inclut également une consommation élevée d'alcool, de produits transformés et de produits à haute densité énergétique, ainsi qu'une faible consommation de fruits et légumes, et qui s'associe à un mode de vie sédentaire. Ainsi, il n'est pas toujours évident de distinguer l'effet propre de la consommation de viande rouge et de charcuterie de celui du régime occidental dans son ensemble (15).

\subsubsection{Fruits et légumes}

Les fruits et légumes ont une faible densité énergétique et un faible index glycémique. Ils sont riches en vitamines, en minéraux, en micronutriments et en fibres. Ainsi, ils apportent un volume important de nourriture, contribuent à l'apport en micronutriments et ont un faible impact sur l'apport énergétique. La consommation de fruits et légumes est associée à une diminution du risque de mortalité par accidents coronariens, par accidents vasculaires cérébraux et par certains cancers (poumon, tube digestif). Les diminutions de risques associées à la consommation des fruits et légumes sont relativement modestes, mais significatives. Elles sont généralement de l'ordre de $5 \%$ par portion supplémentaire de fruits et légumes et de l'ordre de $20 \%$ si l'on compare les catégories extrêmes de consommateurs (21). Comme pour la consommation de viande, il est difficile de distinguer l'effet propre de la consommation des fruits et légumes de celui du régime alimentaire global qui lui est associé. Le lien causal entre la consommation de fruits et légumes et les effets sur la santé peut être discuté. L'effet de la consommation des fruits et légumes sur le niveau de pression artérielle a été démontré dans des études interventionnelles (22) mais, à l'inverse, en ce qui concerne la supplémentation en antioxydants, il n'a pas été retrouvé d'effet dans les études expérimentales (23).

\subsubsection{Autres produits d'origine végétale}

De nombreux autres produits d'origine végétale sont associés à un impact sur la santé. Par exemple, la consommation de produits céréaliers complets, riches en fibres, a été associée à une diminution des facteurs de risque cardiovasculaire, du risque de cardiopathie ischémique, d'accidents vasculaires cérébraux et de cancer du côlon (24).

Les fruits à coques sont des aliments à densité énergétique élevée riches en protéines et en acides gras poly-insaturés (PUFA) et mono-insaturés (MUFA) ainsi qu'en fibres, vitamines, sels minéraux et polyphénols. Malgré leur forte densité énergétique et leur contenu en lipides, la consommation de fruits à coques n'a pas été associée à un risque plus élevé d'obésité. Au contraire, des associations protectrices avec l'indice de masse corporelle (IMC) sont même parfois retrouvées dans certaines études. La consommation de fruits à coques a été retrouvée associée à une diminution du niveau de cholestérol total et de LDL-cholestérol dans des études épidémiologiques et des essais randomisés. En plus de l'effet sur les maladies vasculaires, les noix sont riches en composants neuro-protecteurs (polyphénols, acide alpha-linolénique, mélatonine, folate, vitamine E) et une association positive entre la consommation de fruits à coques ou de noix et le score cognitif a été retrouvée dans des études épidémiologiques (25).

Les légumineuses qui sont utilisées comme sources de protéines chez les végétariens ont des effets potentiels protecteurs contre les maladies cardiovasculaires et les cancers. Les légumineuses sont riches en fibres et apportent des acides gras insaturés, de la vitamine $E$, du folate, du sélénium et 
des flavonoïdes qui ont des effets protecteurs potentiels (26). Cependant, si une diminution des risques cardiovasculaires (27) et des cancers (26) associée à la consommation de légumineuses a été observée dans certaines études de cohorte, il existe des résultats contradictoires provenant d'autres études épidémiologiques $(26,28)$.

\section{Données épidémiologiques}

\subsection{Méthodologie des études}

7 Les données épidémiologiques sur les associations entre alimentation et pathologies chroniques 8 reposent essentiellement sur des études de cohorte prospectives. Dans ces études, l'alimentation des participants est généralement recueillie à l'inclusion. Les participants sont ensuite suivis de nombreuses années. Ces études portent généralement sur des événements de santé (cancers, maladies vasculaires, décès). II faut un nombre d'événements suffisant afin d'obtenir la puissance statistique nécessaire. Généralement, seule une faible proportion des personnes participant à l'étude est victime d'un ou plusieurs de ces événements de santé lors du suivi. Pour cette raison, ces études nécessitent un très grand nombre de participants. II existe de nombreuses études de cohorte dans le monde, disposant d'informations portant sur la nutrition. On peut citer à titre d'exemple les cohortes des professionnels de santé américains constituées dans les années 1980 (Nurses' Health study et Health Professionals' Follow-Up study incluant respectivement 120000 femmes et 50000 hommes (29)). En France, on peut citer les cohortes SU.VI.MAX (SUpplémentation en VItamines et Minéraux Anti-oXydants) (30) et Nutrinet (31). Plus récemment, des cohortes de très grande taille comprenant plusieurs centaines de milliers de sujets ont été constituées (cohortes Constances (32) et UK Biobank $(33,34)$ par exemple). Cependant, la proportion de consommateurs de régimes végétariens (et à plus forte raison celle des végétaliens) restant faible, malgré des échantillons de population de taille très importante, ces études ne permettent pas d'étudier l'association entre la consommation de régimes végétariens et la survenue d'événements de santé. Ainsi, les données épidémiologiques portant sur ce sujet sont plus rares que sur d'autres questions concernant l'alimentation et doivent être recueillies auprès de populations spécifiques.

\subsubsection{Exemples de cohortes étudiant les régimes végétariens}

\subsubsection{Recrutement ciblé de volontaires végétariens}

Les études de cohorte sont généralement constituées d'un échantillon de volontaires recrutés en population générale. Une fois les personnes incluses, elles sont interrogées sur leurs consommations alimentaires. Comme évoqué dans le paragraphe précédent, avec cette méthode, la proportion de végétariens dans l'étude est généralement faible, proche de celle observée dans la population générale. Afin d'augmenter leur effectif, une solution consiste à contacter spécifiquement des personnes adoptant un régime végétarien. C'est ce qui a été fait en particulier dans les deux études de cohorte d'Oxford (35). Les participants végétariens de la première cohorte (Oxford Vegetarian study, 1980-1984) ont été recrutés par annonce dans les médias et par le bouche-à-oreille. Les participants de la deuxième cohorte (EPIC-Oxford cohort study, 1993-1999) ont été recrutés par courrier, parmi les membres de la "Vegetarian Society of the United Kingdom » et un questionnaire dans le magazine de la "Vegan Society ». Un inconvénient de cette méthode est qu'elle nécessite de recruter un échantillon "témoin ", c'est-à-dire des non végétariens afin de permettre une comparaison. Cet échantillon témoin est issu d'une population différente de celle des végétariens, ce qui peut biaiser les résultats. Dans l'Oxford Vegetarian study, les témoins non végétariens ont été recrutés parmi les amis et les proches des végétariens inclus dans l'étude. Dans l'étude EPIC-Oxford cohort study, les témoins ont été recrutés parmi les patientèles de médecins généralistes. Au total, ces deux cohortes ont inclus 62000 personnes dont environ la moitié de végétariens. 


\subsubsection{Choix d'une population où la proportion de végétariens est naturellement élevée}

Une autre possibilité pour augmenter la proportion de végétariens dans les études est d'inclure un échantillon de personnes issues d'une population où, en raison d'habitudes culturelles ou religieuses, la proportion de végétariens est élevée. C'est le choix qui a été fait pour constituer les cohortes des " adventistes ». Dans ces cohortes, des adeptes nord-américains de l'église adventiste ont été inclus. L'église adventiste promeut un style de vie favorable à la santé. En effet, il est attendu des pratiquants d'être non-fumeurs, de ne pas consommer d'alcool et les pratiquants sont encouragés à adopter un régime végétarien. Le suivi de ces recommandations est variable, les adventistes en Amérique du Nord sont majoritairement non-fumeurs et ne consomment pas ou très peu d'alcool. En ce qui concerne le régime alimentaire, environ la moitié d'entre eux ont un régime omnivore conventionnel, les autres ont un régime flexitarien, ovo/lacto-végétarien ou végétalien. Trois cohortes d'adventistes ont été constituées, les deux premières en Californie (Adventist Mortality study (1960-1976) et Adventist Health Study-1 (AHS-1) (1976-1988) incluant 23000 et 34 000 personnes respectivement) et la troisième (Adventist Health Study-2 (AHS-2) avec 96000 inclusions) dans toute l'Amérique du Nord $(8,36)$.

La plupart des données épidémiologiques sur les régimes végétariens proviennent des cohortes d'Oxford et des adventistes. D'autres cohortes ont analysé l'association entre les régimes végétariens et les événements de santé ou la mortalité $(37,38)$, mais leurs effectifs de végétariens étaient moins importants.

\subsection{Principaux résultats}

\subsubsection{Régimes végétariens}

\subsubsection{Indice de masse corporelle (IMC)}

Les régimes végétariens sont associés à un moindre risque d'obésité et de surpoids dans les études des adventistes et dans les études d'Oxford. Dans I'AHS-2, I'IMC moyen était de $28,8 \mathrm{~kg} / \mathrm{m}^{2}$ dans le groupe des omnivores contre $25,7 \mathrm{~kg} / \mathrm{m}^{2}$ chez les ovo/lacto-végétariens. L'IMC était encore plus bas chez les végétaliens $\left(23,6 \mathrm{~kg} / \mathrm{m}^{2}(39)\right)$. Cette même tendance était retrouvée dans les études d'Oxford (35) dans une population européenne avec des IMC moyens plus bas. L'IMC moyen était de $24,5 \mathrm{~kg} / \mathrm{m}^{2}$ chez les omnivores contre $23,2 \mathrm{~kg} / \mathrm{m}^{2}$ chez les végétariens et $22,4 \mathrm{~kg} / \mathrm{m}^{2}$ chez les végétaliens. Dans une méta-analyse, Dinu et al. (40) ont retrouvé un IMC inférieur de $1,72 \mathrm{~kg} / \mathrm{m}^{2}$ en moyenne chez les végétariens.

\subsubsection{Risque cardiovasculaire}

Le régime végétarien est aussi associé à une diminution du risque cardiovasculaire. Dans la métaanalyse de Dinu et al., le régime végétarien était associé à un niveau plus bas de LDL-cholestérol ($22 \mathrm{mg} / \mathrm{dL}$ ) et de cholestérol total $(-31 \mathrm{mg} / \mathrm{dL})$. Dans I'AHS-2, le risque de diabète était presque divisé par deux chez les végétaliens et les lacto/ovo-végétariens et diminué d'environ 25 à $30 \%$ chez les pesco-végétariens et les flexitariens (39).

La méta-analyse de Dinu et al. retrouvait également une diminution significative de $25 \%$ (RR 0,75 $[0,68-0,82])$ du risque d'accident coronarien sur 7 cohortes. Cependant, les associations étaient non significatives pour l'ensemble des accidents cardiovasculaires (RR 0,93 [0,86-1,00] sur 5 cohortes) et pour le risque d'accident cérébro-vasculaire (RR 0,93 [0,78-1,10]) sur 5 cohortes.

\subsubsection{Cancers}

43 Sur l'ensemble des cancers, une diminution de l'incidence d'environ $10 \%$ était observée dans les 44 cohortes d'Oxford parmi les végétariens (RR 0,90 [0,84-0,97]) et les pesco-végétariens (RR 0,89 

pesco-végétariens (RR 0,88 [0,77-1,01]) et les végétariens (RR 0,92 [0,85-0,99]) (41).

Concernant les localisations spécifiques, la méta-analyse de Dinu et al. (40) ne retrouvait pas d'association significative entre le régime végétarien et le risque de cancer du sein ou de cancer de la prostate sur l'ensemble des études. Une diminution significative du risque de mortalité par cancer du sein était observée uniquement dans les cohortes d'adventistes.

\subsubsection{Mortalité}

Les données portant sur l'association entre les régimes végétariens et la mortalité sont contradictoires. Dans la méta-analyse de Dinu et al. (40), une diminution significative de $16 \%$ de la mortalité était observée chez les végétariens des cohortes d'adventistes (RR 0,84 [0,78-0,90] pour 3 cohortes) mais pas chez les non-adventistes (RR 1,04 [0,98-1,10] pour 4 cohortes). Ces différences posent la question de la possibilité d'extrapoler les données des cohortes d'adventistes à une population plus large. Dans les cohortes d'adventistes, les végétariens pourraient également être ceux qui respectent aussi les préceptes favorables à la santé de l'église adventiste, ce qui pourrait expliquer le plus faible taux de mortalité chez les végétariens. A l'inverse, dans les études d'Oxford, les non-végétariens étaient issus d'une population différente de celle des végétariens, ce qui a pu biaiser les résultats et éventuellement masquer une différence entre les groupes.

\subsubsection{Régimes végétaliens}

Si l'on peut s'attendre à retrouver les bénéfices potentiels du régime végétarien chez les végétaliens, la couverture optimale des besoins nutritionnels pourrait être plus difficile à atteindre chez les végétaliens. Il est donc important d'étudier plus spécifiquement les régimes végétaliens. Cependant, la proportion de végétaliens dans la population est très faible. Même dans les cohortes ayant un recrutement ciblé telles que les cohortes d'Oxford et des adventistes, leurs effectifs restent faibles. Pour cette raison, les données sont rares. Les études d'Oxford et l'AHS-2 retrouvaient une diminution du risque de cancer légèrement supérieure chez les végétaliens par rapport aux végétariens, avec un risque plus faible de $19 \%$ dans les études d'Oxford (RR à $0,81[0,83-0,96])(35)$ et de $16 \%$ (RR 0,84 $[0,72-0,99])(41)$ dans les études des adventistes. Dans la méta-analyse de Dinu et al. (40), sur un effectif réduit de 2 études et 6301 sujets, la mortalité était diminuée de 12\% (RR 0,88 $[0,75-1,02])$ chez les végétaliens. Ce résultat n'était pas significatif en raison de la petite taille de l'effectif. Ces rares résultats sur le cancer et la mortalité suggèrent un bénéfice du régime végétalien bien conduit par rapport à un régime omnivore en ce qui concerne le risque de maladies chroniques et la mortalité chez l'adulte.

\subsection{Limite des études épidémiologiques de cohortes pour l'évaluation du régime végétarien}

Les études épidémiologiques retrouvent donc, généralement, que les végétariens présentent une incidence plus faible de maladies chroniques (cancers, maladies cardiovasculaires, obésité) comparés aux personnes ayant un régime omnivore conventionnel. Néanmoins, la faible proportion de la population consommant un régime végétarien nécessite d'avoir recours à des méthodes de recrutement spécifiques. Les végétariens sont majoritairement recrutés parmi la communauté adventiste ou par l'intermédiaire d'associations ou de revues spécialisées (études d'Oxford). Les végétariens et végétaliens inclus dans ces études sont donc probablement bien informés et ont probablement, majoritairement, un régime bien conduit couvrant l'ensemble des besoins nutritionnels. D'autre part, ces personnes adoptant un régime végétarien ont probablement d'autres comportements bénéfiques pour la santé. Dans l'étude d'Oxford, les végétariens " bien informés " 
étaient comparés à des personnes recrutées dans les cabinets de médecine générale, qui ne prêtaient probablement pas plus attention à leur alimentation que la population générale, ce qui a pu introduire un biais en faveur des régimes végétariens. Les résultats des études épidémiologiques actuelles ne peuvent donc être extrapolés qu'aux régimes végétariens et végétaliens bien conduits chez des personnes informées.

A l'inverse, les personnes débutant un régime végétarien sans appartenir à une communauté, sans information et sans suivi sont probablement difficilement incluses dans ces études. Ce sont ces populations qui sont les plus à risque d'apports nutritionnels insuffisants. II serait donc nécessaire d'avoir plus d'informations sur ces groupes à risque.

\subsection{Régime végétarien et qualité de l'alimentation}

Les études des adventistes ont été conduites en Amérique du Nord et les études d'Oxford en Royaume-Uni. Il existe peu de données issues d'autres pays. Dans ces études, les personnes ayant un régime végétarien sont donc comparées à une population ayant une alimentation généralement de type occidental (western diet). Ainsi, par exemple, dans un échantillon représentatif de la population américaine de l'étude NHANES (2007-2012), la qualité de l'alimentation mesurée par le score Healthy Eating Indes (HEI) était très supérieure chez les personnes ayant un régime végétarien par rapport aux consommateurs de viande. En effet, $45 \%$ des végétariens étaient dans le quintile le plus élevé de qualité alimentaire contre $20 \%$ des consommateurs de viande (42). Cette association a été retrouvée dans d'autres études par exemple en France (31) et en Belgique (43). Le régime végétarien est donc généralement comparé dans les études épidémiologiques à des régimes de qualité nutritionnelle moindre et le bénéfice propre au régime végétarien est difficile à évaluer. Il serait intéressant de comparer les régimes végétariens à une alimentation omnivore de qualité ou à un régime méditerranéen par exemple.

\subsubsection{Bénéfices d'une alimentation végétale (de bonne qualité)}

Les personnes consommant un régime végétarien ont généralement un régime alimentaire de bonne qualité, mais le régime végétarien ou une alimentation riche en produits d'origine végétale n'est pas synonyme d'alimentation bénéfique pour la santé. II est nécessaire pour les régimes végétariens comme pour les régimes omnivores d'avoir une bonne qualité nutritionnelle. Ainsi, par exemple, dans la cohorte des professionnels de santé, Satija et al. (44) ont étudié l'association entre une alimentation riche en produits d'origine végétale et le risque de survenue d'accident coronarien à l'aide de trois scores. Le premier comptabilisait la quantité de produits d'origine végétale dans l'alimentation quelle que soit leur qualité nutritionnelle, le second comptabilisait la quantité de produits d'origine végétale de bonne qualité nutritionnelle (attribution de valeur négative aux aliments d'origine végétale de moins bonne qualité nutritionnelle tels que les jus de fruits, boissons sucrées, frites, friandises, céréales raffinées, ...) et le dernier comptabilisait la quantité de produits d'origine végétale de "mauvaise qualité nutritionnelle». Le premier score était associé à une diminution du risque d'accidents coronariens. Cette association était encore plus forte lorsqu'on excluait les produits végétaux de moins bonne qualité nutritionnelle. A l'inverse, le risque d'accident coronarien était fortement augmenté lorsque les scores d'alimentation d'origine végétale de moins bonne qualité étaient élevés. Ces résultats suggèrent que, si en général une végétalisation de I'alimentation améliore la qualité de l'alimentation et la santé, c'est à condition que les aliments d'origine végétale choisis soient de bonne qualité nutritionnelle. 
2 Les régimes végétariens sont donc généralement associés à une meilleure santé, un moindre risque 3 de cancers, de maladies cardiovasculaires et de mortalité dans les études épidémiologiques. Ces 4 études suggèrent un bénéfice pour la santé des régimes végétariens. Peu de donnés spécifiques sont 5 actuellement disponibles sur les régimes végétaliens mais il semble qu'ils soient également associés 6 à un bénéfice pour la santé. Cependant, ces résultats sont obtenus dans des populations d'adultes 7 d'âges moyens et ne sont pas nécessairement extrapolables aux populations fragiles telles que les 8 personnes âgées, les enfants et les adolescents en période de croissance. De par leur mode de 9 recrutement, ces études incluent majoritairement des personnes bien informées, les risques 10 d'insuffisance d'apports liés notamment à un régime végétalien mal conduit y sont donc mal 11 évalués. Des risques de malnutrition liés en particulier à l'engouement récent pour les régimes végétaliens sont possibles et devraient faire l'objet d'une surveillance sanitaire. Enfin, dans les études épidémiologiques, les personnes adoptant des régimes végétariens sont généralement comparées à des groupes de personnes ayant une alimentation de qualité moyenne. II serait intéressant de comparer le bénéfice apporté par un régime végétarien à celui d'un régime omnivore bien équilibré.

\section{Conclusion}

Les régimes végétariens peuvent comporter des risques d'insuffisance d'apports nutritionnels s'ils sont mal conduits, tandis que s'ils sont bien menés ils sont généralement associés à des bénéfices en termes de santé. L'engouement récent pour les régimes végétaliens, en particulier, nécessite de rester vigilant sur les risques des régimes végétaliens mal conduits. Même si la proportion de la population ayant un régime végétarien reste faible, il est probable que la consommation de produits d'origine animale diminue chez les personnes ayant un régime omnivore conventionnel en raison des modifications des recommandations et de considérations environnementales. Cette évolution et son impact sur la santé seront à évaluer dans les futures études épidémiologiques. 
Références bibliographiques

1. Mariotti F, editor. Vegetarian and plant-based diets in health and disease prevention. London San Diego Cambridge Kidlington: Elsevier, Academic Press; 2017. 887 p.

2. Dagnelie PC, Mariotti F. Vegetarian Diets. In: Vegetarian and Plant-Based Diets in Health and Disease Prevention [Internet]. Elsevier; 2017 [cited 2019 Apr 29]. p. 3-10. Available from: https://linkinghub.elsevier.com/retrieve/pii/B9780128039687000010

3. Avis de l'Anses, Avis de l'Anses,Rapport d'expertise collective. Étude individuelle nationale des consommations alimentaires 3 (INCA 3). 2017 Juin.

4. Kim H, Rotundo L, Song D, Demyen M, Ahlawat S. The Prevalence and Characteristics of Vegetarian in the United States: A Population-Based Study. Gastroenterology. 2017 Apr;152(5):S1016.

5. Gert B.M. Mensink, Clarissa Lage Barbosa, Robert Koch-Institut. Prevalence of persons following a vegetarian diet in Germany. RKI-Bib1 Robert Koch-Inst [Internet]. 2016 [cited 2019 Apr 29]; Available from: http://edoc.rki.de/docviews/abstract.php?lang=ger\&id=4888

6. Agrawal S. Geographic Aspects of Vegetarianism. In: Vegetarian and Plant-Based Diets in Health and Disease Prevention [Internet]. Elsevier; 2017 [cited 2019 Apr 29]. p. 93-106. Available from: https://linkinghub.elsevier.com/retrieve/pii/B978012803968700006X

7. Arnold F, Parasuraman S, Arokiasamy $\mathrm{P}$, Kothari M. Nutrition in india national family health survey. in NFH>S3. 2005-06 Mumbai : interntional institute for Population Sciences Vo. I?ICF Macro, Calverton, Maryland, USA. 2009.

8. Le L, Sabaté J. Beyond Meatless, the Health Effects of Vegan Diets: Findings from the Adventist Cohorts. Nutrients. 2014 May 27;6(6):2131-47.

9. Position Paper on the vegetarian approach to eating. J Am Diet Assoc. 1980 Jul;77(1):61-9.

10. Havala S, Dwyer J. Position of the American Dietetic Association: vegetarian diets. J Am Diet Assoc. 1993 Nov;93(11):1317-9.

11. Melina V, Craig W, Levin S. Position of the Academy of Nutrition and Dietetics: Vegetarian Diets. J Acad Nutr Diet. 2016 Dec;116(12):1970-80.

12. Haut Conseil de la santé publique. Avis relatif à la révision des repères alimentaires pour les adultes du futur Programme national nutrition santé 2017-2021. 2017.

13. Mariotti F. Plant Protein, Animal Protein, and Protein Quality. In: Vegetarian and Plant-Based Diets in Health and Disease Prevention [Internet]. Elsevier; 2017 [cited 2019 Apr 29]. p. 62142. Available from: https://linkinghub.elsevier.com/retrieve/pii/B9780128039687000356

14. Appleby $\mathrm{P}$, Roddam $\mathrm{A}$, Allen $\mathrm{N}$, Key $\mathrm{T}$. Comparative fracture risk in vegetarians and nonvegetarians in EPIC-Oxford. Eur J Clin Nutr. 2007 Dec;61(12):1400-6.

15. Boutron-Ruault M-C, Mesrine S, Pierre F. Meat Consumption and Health Outcomes. In: Vegetarian and Plant-Based Diets in Health and Disease Prevention [Internet]. Elsevier; 2017 [cited 2019 Apr 29]. p. 197-214. Available from: https://linkinghub.elsevier.com/retrieve/pii/B9780128039687000125 
16. Bouvard V, Loomis D, Guyton KZ, Grosse Y, Ghissassi FE, Benbrahim-Tallaa L, et al. Carcinogenicity of consumption of red and processed meat. Lancet Oncol. 2015 Dec;16(16):1599-600.

17. O'Sullivan TA, Hafekost K, Mitrou F, Lawrence D. Food sources of saturated fat and the association with mortality: a meta-analysis. Am J Public Health. 2013 Sep;103(9):e31-42.

18. Alexander DD, Weed DL, Cushing CA, Lowe KA. Meta-analysis of prospective studies of red meat consumption and colorectal cancer. Eur J Cancer Prev Off J Eur Cancer Prev Organ ECP. 2011 Jul;20(4):293-307.

19. Feskens EJM, Sluik D, van Woudenbergh GJ. Meat consumption, diabetes, and its complications. Curr Diab Rep. 2013 Apr;13(2):298-306.

20. Abete I, Romaguera D, Vieira AR, Lopez de Munain A, Norat T. Association between total, processed, red and white meat consumption and all-cause, CVD and IHD mortality: a metaanalysis of cohort studies. Br J Nutr. 2014 Sep 14;112(5):762-75.

21. van Berleere M, Dauchet L. Fruits, Vegetables, and Health. In: Vegetarian and Plant-Based Diets in Health and Disease Prevention [Internet]. Elsevier; 2017 [cited 2019 Apr 29]. p. 215-48. Available from: https://linkinghub.elsevier.com/retrieve/pii/B9780128039687000137

22. Appel L, Moore TJ, Obarzanek E, Vollmer WM, Svetkey LP, Sacks FM, et al. A Clinical Trial of the Effects of Dietary Patterns on Blood Pressure. N Engl J Med. 1997 Apr 17;336(16):1117-24.

23. Bjelakovic G, Nikolova D, Gluud C. Antioxidant supplements and mortality: Curr Opin Clin Nutr Metab Care. 2013 Nov;1.

24. Thies F. Whole Grains and Disease Risk. In: Vegetarian and Plant-Based Diets in Health and Disease Prevention [Internet]. Elsevier; 2017 [cited 2019 Apr 29]. p. 249-69. Available from: https://linkinghub.elsevier.com/retrieve/pii/B9780128039687000149

25. Wien M. Nut Intake and Health. In: Vegetarian and Plant-Based Diets in Health and Disease Prevention [Internet]. Elsevier; 2017 [cited 2019 Apr 29]. p. 271-92. Available from: https://linkinghub.elsevier.com/retrieve/pii/B9780128039687000150

26. Papandreou C, Becerra-Tomás N, Bulló M, Martínez-González MÁ, Corella D, Estruch R, et al. Legume consumption and risk of all-cause, cardiovascular, and cancer mortality in the PREDIMED study. Clin Nutr Edinb Scotl. 2019 Feb;38(1):348-56.

27. Nöthlings $U$, Schulze MB, Weikert $C$, Boeing $H$, van der Schouw $Y T$, Bamia C, et al. Intake of vegetables, legumes, and fruit, and risk for all-cause, cardiovascular, and cancer mortality in a European diabetic population. J Nutr. 2008 Apr;138(4):775-81.

28. Miller V, Mente A, Dehghan M, Rangarajan S, Zhang X, Swaminathan S, et al. Fruit, vegetable, and legume intake, and cardiovascular disease and deaths in 18 countries (PURE): a prospective cohort study. Lancet Lond Engl. 2017 Nov 4;390(10107):2037-49.

29. Joshipura KJ, Hu FB, Manson JE, Stampfer MJ, Rimm EB, Speizer FE, et al. The Effect of Fruit and Vegetable Intake on Risk for Coronary Heart Disease. Ann Intern Med. 2001 Jun 19;134(12):1106. 
30. Hercberg S, Galan P, Preziosi P, Bertrais S, Mennen L, Malvy D, et al. The SU.VI.MAX Study: a randomized, placebo-controlled trial of the health effects of antioxidant vitamins and minerals. Arch Intern Med. 2004 Nov 22;164(21):2335-42.

31. Allès B, Baudry J, Méjean C, Touvier M, Péneau S, Hercberg S, et al. Comparison of Sociodemographic and Nutritional Characteristics between Self-Reported Vegetarians, Vegans, and Meat-Eaters from the NutriNet-Santé Study. Nutrients. 2017 Sep 15;9(9):1023.

32. http://www.constances.fr/ accédé le 29/04/2019.

33. Sudlow C, Gallacher J, Allen N, Beral V, Burton P, Danesh J, et al. UK Biobank: An Open Access Resource for Identifying the Causes of a Wide Range of Complex Diseases of Middle and Old Age. PLOS Med. 2015 Mar 31;12(3):e1001779.

34. Anderson JJ, Darwis NDM, Mackay DF, Celis-Morales CA, Lyall DM, Sattar N, et al. Red and processed meat consumption and breast cancer: UK Biobank cohort study and meta-analysis. Eur J Cancer. 2018 Feb;90:73-82.

35. Key TJ, Appleby PN, Crowe FL, Bradbury KE, Schmidt JA, Travis RC. Cancer in British vegetarians: updated analyses of 4998 incident cancers in a cohort of 32,491 meat eaters, 8612 fish eaters, 18,298 vegetarians, and 2246 vegans. Am J Clin Nutr. 2014 Jul;100 Suppl 1:378S-85S.

36. Butler TL, Fraser GE, Beeson WL, Knutsen SF, Herring RP, Chan J, et al. Cohort Profile: The Adventist Health Study-2 (AHS-2). Int J Epidemiol. 2008 Apr;37(2):260-5.

37. Chang-Claude J. Lifestyle Determinants and Mortality in German Vegetarians and HealthConscious Persons: Results of a 21-Year Follow-up. Cancer Epidemiol Biomarkers Prev. 2005 Apr 1;14(4):963-8.

38. Cade JE, Taylor EF, Burley VJ, Greenwood DC. Common Dietary Patterns and Risk of Breast Cancer: Analysis From the United Kingdom Women's Cohort Study. Nutr Cancer. 2010 Apr 5;62(3):300-6.

39. Tonstad S, Butler T, Yan R, Fraser GE. Type of vegetarian diet, body weight, and prevalence of type 2 diabetes. Diabetes Care. 2009 May;32(5):791-6.

40. Dinu M, Abbate R, Gensini GF, Casini A, Sofi F. Vegetarian, vegan diets and multiple health outcomes: A systematic review with meta-analysis of observational studies. Crit Rev Food Sci Nutr. 2017 Nov 22;57(17):3640-9.

41. Tantamango-Bartley $Y$, Jaceldo-SiegI K, Fan J, Fraser G. Vegetarian diets and the incidence of cancer in a low-risk population. Cancer Epidemiol Biomark Prev Publ Am Assoc Cancer Res Cosponsored Am Soc Prev Oncol. 2013 Feb;22(2):286-94.

42. Conrad Z, Karlsen M, Chui K, Jahns L. Diet quality on meatless days: National Health and Nutrition Examination Survey (NHANES), 2007-2012. Public Health Nutr. 2017 Jun;20(9):156473.

43. Clarys $P$, Deliens $T$, Huybrechts I, Deriemaeker P, Vanaelst B, De Keyzer W, et al. Comparison of nutritional quality of the vegan, vegetarian, semi-vegetarian, pesco-vegetarian and omnivorous diet. Nutrients. 2014 Mar 24;6(3):1318-32. 
1

44. Satija A, Bhupathiraju SN, Spiegelman D, Chiuve SE, Manson JE, Willett W, et al. Healthful and Unhealthful Plant-Based Diets and the Risk of Coronary Heart Disease in U.S. Adults. J Am Coll Cardiol. 2017 Jul 25;70(4):411-22. 


\section{Remerciement}

2 Remerciement à Brigitte Bonneau pour sa relecture. 